\title{
Saccadic Suppression of Retinotopically Localized Blood Oxygen Level-Dependent Responses in Human Primary Visual Area V1
}

\author{
Ignacio Vallines ${ }^{1,2}$ and Mark W. Greenlee ${ }^{1}$ \\ ${ }^{1}$ Department of Experimental Psychology, University of Regensburg, D-93053 Regensburg, Germany, and 2Department of Experimental Psychology, \\ Ludwig-Maximilians-University, D-80802 Munich, Germany
}

\begin{abstract}
Saccadic eye movements are responsible for bringing relevant parts of the visual field onto the fovea for detailed analysis. Because the retina is physiologically unable to deliver sharp images at very high transsaccadic speeds, the visual system minimizes the repercussion of the blurry images we would otherwise perceive during transsaccadic vision by reducing general visual sensitivity and increasing the detection threshold for visual stimuli. Ruling out a pure retinal origin, the effects of saccadic suppression can be already observed some $75 \mathrm{~ms}$ before the onset of a saccadic eye movement and are maximal at the onset of motion. The perception of a briefly presented stimulus immediately before the onset of any retinal motion is thus impaired despite the fact that this stimulus is projected onto the stationary retina and is, therefore, physically identical to that presented when no saccadic programming is in course. In this functional magnetic resonance imaging event-related study, we flashed Gabor patches at different times before the onset of a horizontal saccade and measured blood oxygen level-dependent responses at their encoding regions in primary visual cortex (V1) while subjects judged the relative orientation of the stimuli. Closely matching the significant reduction in behavioral performance, the amplitude of the responses in V1 consistently decreased as the stimuli were presented closer to the saccadic onset. These results demonstrate that the neural processes underlying saccade programming transiently modulate cortical responses to briefly presented visual stimuli in areas as early a V1, providing additional evidence for the existence of an active saccadic suppression mechanism in humans.
\end{abstract}

Key words: saccade; vision; cortex; eye movement; fMRI; striate cortex; saccadic suppression; V1; retinotopy

\section{Introduction}

Vision is an active process involving a close interplay between sensory and oculomotor control systems in the brain. Although the existence of an active saccadic suppression mechanism and its putative extraretinal origin has been the subject of intense debate (Castet et al., 2001; Garcia-Perez and Peli, 2001; Ross et al., 2001), it is generally accepted that low-spatial-frequency luminancedefined stimuli, which are extrafoveally presented and thus mainly processed by the magnocellular pathway, are most suppressed during saccades (Burr and Morrone, 1994).

Although previous imaging studies have reported a decrease of activity in primary visual cortex (V1) related to saccade frequency (Paus et al., 1995; Wenzel et al., 2000), first attempts to couple stimulus onset information and oculomotor measurements in an event-related functional magnetic resonance imaging (fMRI) experiment found correlates of saccadic suppression to luminance-defined stimuli (but not for isoluminant chromatically defined stimuli) in the dorsal stream [V5/hMT+ (human motion complex), V7] and in ventral area V4 (Kleiser et al.,

Received 0ct. 6, 2005; revised April 21, 2006; accepted April 21, 2006.

This work was supported by Deutsche Forschungsgemeinschaft (DFG) Sonderforschungsbereich 517 Neurokognition (project C9) and the DFG International Graduate School for Neurosensory Science and Systems at the Carl von Ossietzky University in Oldenburg.

Correspondence should be addressed to Ignacio Vallines, Department of Experimental Psychology, LudwigMaximilians-University, Leopoldstrasse 13, D-80802 Munich, Germany. E-mail: vallines@Imu.de.

DOI:10.1523/JNEUROSCI.0817-06.2006

Copyright $\odot 2006$ Society for Neuroscience $\quad$ 0270-6474/06/265965-05\$15.00/0
2004). These authors concluded that saccadic suppression occurs at higher motion-sensitive areas and found no significant evidence of saccadic suppression in areas V1 and V2. Thilo et al. (2004) reported that saccades impair the perception of phosphenes elicited at the retinal level but not of those elicited by transcranial magnetic stimulation over the occipital cortex, suggesting that retinal signals must be suppressed before arriving at the visual cortex. Suppression at an early stage of visual processing is also supported by fMRI work from Sylvester et al. (2005) in which they demonstrate that sequences of saccades during ganzfeld visual stimulation modulate activity in human lateral geniculate nucleus (LGN), V1, and V2.

Well established psychophysical research has shown that saccadic suppression provokes a transient and short-lived decrease in visual sensitivity that begins $\sim 75 \mathrm{~ms}$ before the onset of the actual eye movement and is maximal at motion onset (Latour, 1962; Zuber and Stark, 1966; Volkmann et al., 1968; Riggs et al., 1982; Diamond et al., 2000). The perception of stimuli presented very briefly during this presaccadic interval is impaired despite the fact that they are projected onto a stationary retina, and therefore their retinal images are identical to the ones projected by the same stimuli when no saccadic programming is in course. Right until the moment in which the eyes start to move, retinotopic correspondence is maintained and the encoding location of the visual stimulus can be accurately localized in primary visual cortex (Engel et al., 1997).

In this study, we measured blood oxygen level-dependent 
(BOLD) (Ogawa et al., 1990) responses to four retinotopically localized low-spatialfrequency Gabor stimuli flashed immediately before the onset of a horizontal eye movement to a visual target: the precise moment in which both retinas are still stationary, the encoding location of the stimuli in V1 is known, and saccadic suppression is thought to be maximal. If saccadic suppression modulates signals in primary visual cortex, we would expect BOLD responses elicited by these stimuli to be affected by the temporal proximity to the saccadic onset, the same way performance drops in detection and discrimination tasks.

\section{Materials and Methods}

Subjects. Each of the four naive participants (three males; age range, 23-26 years) gave informed consent and participated in two training sessions outside of the MRI scanner, during which they became acquainted with the task, the eye movement quality was assessed, and a psychometric function for the orientation discrimination was acquired. During the first fMRI session, four measurements were performed to retinotopically localize the encoding location of the four Gabors in V1 and to ensure the detectability of changes in BOLD signal elicited by briefly flashed ( $8 \mathrm{~ms}$ ) Gabor stimuli (supplemental Fig. 1, available at www.jneurosci. org as supplemental material). For the main experiment, to obtain a sufficient number of events for the analysis, 12 fMRI measurements distributed over different days were conducted for each subject.

Retinotopic localization of the stimuli in V1. Brain clusters encoding the Gabor stimuli were retinotopically localized by presenting one flickering dartboard ( $6^{\circ}$ in size; maximum contrast; $8 \mathrm{~Hz}$ flicker rate) (Fig. 1) in each of the four locations in which Gabors were subsequently presented in the main experiment. Each single position was presented 10 times for a period of $13.2 \mathrm{~s}$ [echo planar imaging (EPI) sequence parameters and preprocessing identical as in the main experiment] in a block design such that alternating hemispheres were stimulated in the following order: top left, bottom right, bottom left, and top right (Fig. 1). The onset vector for each dartboard on each of the four positions was convolved with a canonical hemodynamic response function (HRF) to set up four regressors for the SPM analysis (see below, Stimuli and procedure). $t$ test contrast vectors were defined for each regressor against all other three to detect voxels that significantly responded to one position but not the others. Clusters exceeding 5 voxels in size and with false discovery rate corrected $p$ values smaller than $0.00001(t>7)$ were defined as regions of interest (ROIs) (Fig. 1). Each subject completed three additional fMRI sessions in which only G trials (see below, Stimuli and procedure) were presented to confirm the detectability of BOLD changes elicited by the flashed Gabor (supplemental Fig. 1, available at www.jneurosci.org as supplemental material) in the time series extracted from the localized ROIs (Fig. 1).

Stimuli and procedure. In an event-related fMRI design, four experimental conditions were randomly interleaved: with variable intertrial intervals of $\sim 9 \mathrm{~s}$, subjects were trained to maintain fixation ( $\mathrm{F}$ trial; $\sim 10 \%$ of the trials), to rapidly execute a saccadic eye movement to an eccentrically appearing target ( $\mathrm{S}$ trial; $\sim 10 \%$ ), to perform a suprathreshold orientation discrimination of four flashed Gabor stimuli while maintaining fixation ( $\mathrm{G}$ trial; $\sim 10 \%$ ), or to perform a saccade and discriminate the orientation of the flashed stimuli, which were presented after the target onset but immediately before the saccadic onset $(\mathrm{G}+\mathrm{S}$ trial; $\sim 70 \%$ ) (Fig. 2). Button responses to the $G+S$ trials resulted from two concurrent judgments: a detection task in which participants were requested to press one of two buttons to signal whether they detected the flashed Gabors (conditions F and S were identical to $G$ and $G+S$ except in that no Gabors were presented), and an orientation discrimination task in which the presence of one slightly tilted Gabor among the four was reported by pressing the right button (oddball present) or left button (no oddball present). A small $\left(0.2^{\circ}\right)$ red dot was used as the saccadic target and was randomly presented along the horizontal meridian $10^{\circ}$ to the left or to the right of the fixation dot. Luminance-defined (maximum contrast) Gabor stimuli were $6^{\circ}$ in diameter [full-width half-maximum (FWHM) of the Gaussian envelope], had a spatial frequency of 1 cycle $/^{\circ}$, and were located in each of the four visual field quadrants $20^{\circ}$ apart horizontally and $12^{\circ}$ apart vertically. Retinal persistence was minimized by the edgeless structure of Gabor stimuli, whose mean luminance is identical to background luminance. Because horizontally and vertically oriented gratings have been shown to elicit equally strong BOLD responses in V1 (Furmanski and Engel, 2000), Gabor stimuli in conditions $\mathrm{G}$ and $\mathrm{G}+\mathrm{S}$ were randomly presented with base orientations of either 0 or $90^{\circ}$. Orientation differences in the discrimination task were individually set during a previous session for each of the participants to reach accuracy levels of $80 \%$ in the G condition (supplemental Fig. 2, available at www.jneurosci.org as supplemental material). Rotation values obtained for the different participants were randomly added or subtracted to the base orientation in the main experiment whenever a deviant Gabor was presented.

Eye movement recording and stimulus presentation. Visual stimulation was created on a visual stimulus generator (VSG2/5) equipped with full frame control and a real-time dedicated central processing unit (Cambridge Research Systems, Rochester, UK). Horizontal eye movements were recorded using a limbus-reflection-based MR-compatible eye tracker (Cambridge Research Systems), digitized onboard at $1 \mathrm{kHz}$, and stored along with stimulus onsets and MR triggers to ensure timing accuracy. Stimulus presentation was rear-projected through a waveguide by a gamma-corrected D-ILA G15U JVC projector (Victor Company of 


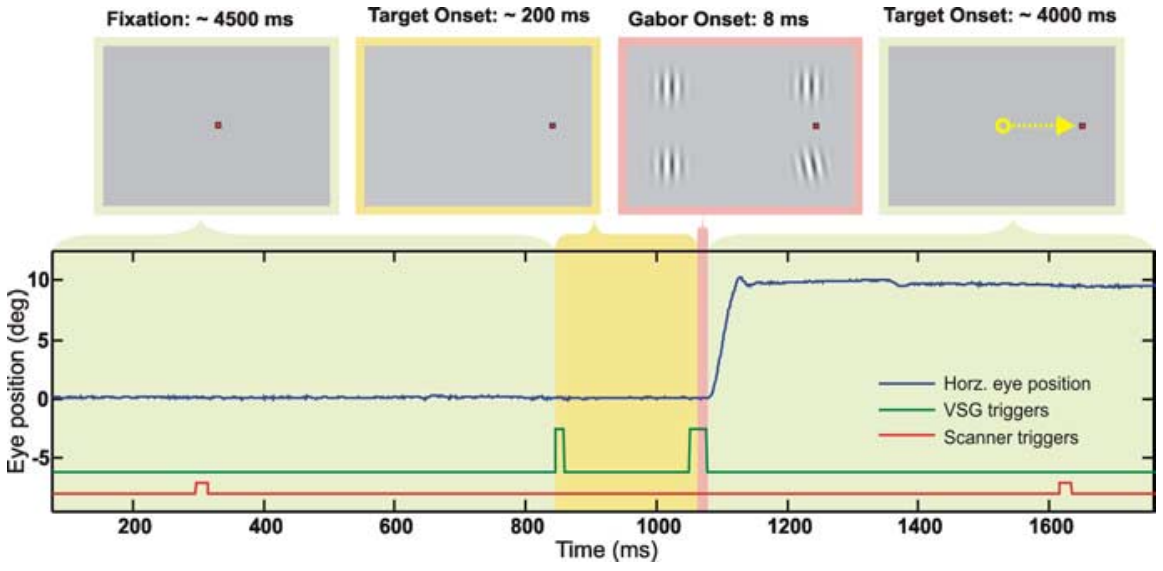

Figure 2. Schematic illustration of the time course of an actual trial from the $G+S$ condition in which Gabor stimuli were flashed immediately before the saccadic onset. At the start of each trial, the participants fixed their gaze on a centrally located fixation dot. In a step procedure, the central fixation dot was extinguished and the saccadic target appeared on the periphery, eliciting the preparation of a saccade. Immediately before saccadic onset, four Gabor stimuli were simultaneously flashed. The bottom represents an actual eye-movement trace. Time marks designate triggers to the scanner acquisition onsets (red) and stimulus onset triggers coming from the visual stimulus generator (green), to which fixed image-formation delays were applied (see Materials and Methods, Eye movement recording and stimulus presentation).

Japan, Yokohama, Japan) positioned outside the RF-shielded scanner room, at a spatial resolution of $1024 \times 768$ pixels and a non-interlaced refresh rate of $78 \mathrm{~Hz}$. A fast photovoltaic transducer was used to measure onset delay, buildup, and decay times in image formation directly on the projection screen. With respect to the FWHM of the temporal luminance profile, we measured a constant delay of $17 \mathrm{~ms}$ and a stimulus duration of 8 ms (supplemental Fig. 3, available at www.jneurosci.org as supplemental material). These values were used to calculate stimulus onset asynchronies (SOAs) by applying them to presentation times marked by digital triggers delivered by the VSG.

An on-line algorithm detected the onset of the saccadic eye movement immediately after the end of each trial by selectively back-reading the recorded eye traces and applying a simple amplitude-velocity thresholding procedure that was optimized for each measurement. A new distribution of the saccadic latencies from previous trials was created during each fixation period, and the optimal Gabor onset time was obtained by taking the modal value of the current binned distribution minus half the duration of the premotoric saccadic suppression reported in the literature $(75 \mathrm{~ms})$. This method considerably improved the probability of the Gabor stimuli to be presented immediately before the onset of the saccadic eye movement (Fig. 2). Only trials in which Gabors were presented immediately before the saccadic onset (in the absence of retinal motion) were included in the analysis.

Stimulus presentation, eye-tracker calibration and eye-movement recordings, trigger management, button-response acquisition, and the online saccadic detection algorithm used to predict the stimulus onset were all implemented in software especially developed for this study. The code was written in Delphi-Pascal by using the VSG version 6 software library.

MRI acquisition. Imaging was performed on a 1.5 tesla Siemens Sonata Maestro (Siemens, Erlangen, Germany), equipped with $40 \mathrm{mT} / \mathrm{m}$ gradients and a high-resolution eight-channel head coil (MRI Devices, Gainesville, FL). Before each measurement, a localizer sequence with seven sagittal slices was used to carefully place eight transversally oriented slices $(3 \times 3 \times 3 \mathrm{~mm}$ voxel size, no gap) along the calcarine fissure of each subject. Each fMRI measurement consisted of 400 such volumes acquired with a Maxwell-corrected EPI sequence running Siemens Integrated Parallel Acquisition Technique (repetition time, 1320 ms; echo time, $77 \mathrm{~ms}$; flip angle, 90; field of view, $192 \mathrm{~mm}$; matrix size, $64 \times 64$; interleaved acquisition). A high spatial resolution (with $1 \mathrm{~mm}$ isotropic voxels) T1-weighted scan [magnetization-prepared rapid-acquisition gradient echo (MPRAGE)] was acquired after each scan session mainly for coregistration purposes.

Data analysis. Saccadic onsets were recalculated off-line by using a set of scripts written in Matlab (MathWorks, Natick, MA) that contained a more sensitive algorithm that included information about the slope of the main sequence obtained from each run. For purposes of quality control, all detected saccadic onsets from all trials were visually inspected by plotting them recursively over the trial period ranging from $100 \mathrm{~ms}$ before and after the detected onset point (supplemental Fig. 4, available at www.jneurosci.org as supplemental material). Saccadic target and Gabor onset vectors were calculated from the VSG digital channels by adding $17 \mathrm{~ms}$ to their raising edges to adjust for the delays introduced by the digital signal transformation within the projector. Gabor onset vector was shifted by $8 \mathrm{~ms}$ to account for their effective onset duration. SOA was then calculated by subtracting the saccadic onset from the corrected offset of the Gabor stimuli so that, in trials with negative SOAs, the stimuli were always flashed before the onset of the eye movement. Only these presaccadic trials were included in the analysis.

Functional images were time and motion corrected, unwarped, coregistered against a high resolution $(1 \times 1 \times 1 \mathrm{~mm}$ voxel size $)$ MPRAGE T1 image, and normalized to a standard template from the Montreal Institute of Neurology (MNI152 T1). Because regions of interest had been precisely localized for each subject, no Gaussian spatial smoothing was applied. Instead, voxel values extracted from the four clusters retinotopically encoding the Gabors were averaged into a single time course. Data analysis was performed at a single subject level based on the general linear model as implemented in the statistical parametric mapping (Friston et al., 1995) package SPM2 (http://www.fil.ion.ucl.ac.uk/spm/software/ spm2/) and used by the region of interest toolbox (Brett et al., 2002) MARSBAR (http://marsbar.sourceforge.net/). Data were global-mean centered, high-pass filtered (cutoff, $0.0078 \mathrm{~Hz}$ ) to remove slow signal drifts, and corrected for intrinsic serial autocorrelations. The five regressors introduced in the design were set up by convolving the onset vectors for F, G, S, and G+S trials and return saccades with a canonical $\mathrm{HRF}$ together with their respective time and dispersion derivatives. In the $\mathrm{G}+\mathrm{S}$ condition, SOAs were introduced as a nonlinear parametric modulator to allow the amplitude of the hemodynamic response function fitted to the data to change as a function of the temporal distance between the presentation of the Gabors and the onset of the saccade. The influence of the parametric modulator was modeled by a second-order polynomial function, and a $t$ test was used to asses whether its introduction significantly explained additional variance. The significance of the variance explained by the $\mathrm{G}+\mathrm{S}$ regressor modulated by $\mathrm{SOA}$, relative to error (goodness of the regression) was estimated by using $F$ statistics (Büchel et al., 1996, 1998).

\section{Results}

Psychometric functions reflecting each subject's sensitivity to the presence of a deviant (oddball) Gabor yielded rotation values leading to $80 \%$ correct responses ranging between 6.6 and $13.7^{\circ}$ for the different subjects (mean, $\pm 9.78 ; \mathrm{SD}, \pm 3.37$ ) (supplemental Fig. 1, available at www.jneurosci.org as supplemental material).

By using the saccadic latency information collected from previous trials, $47.3 \%$ of the Gabor stimuli were presented immediately before the saccadic onset (Fig. 3). Behavioral data show that, even before any retinal motion had begun, subjects failed to detect the stimuli on up to $30 \%$ of the trials in which Gabors were presented immediately before the saccadic onset (i.e., with short negative SOA) (Fig. 3), mistaking a $G+S$ trial for an $S$ trial and therefore giving no button response. Performance in the orientation discrimination task for trials in which Gabor stimuli were detected immediately before the saccadic onset dropped from 82 


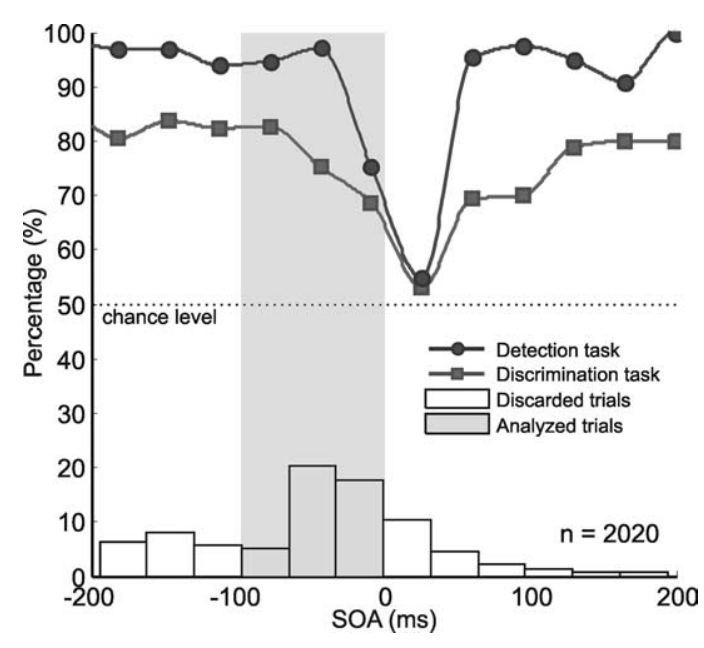

Figure 3. Behavioral cumulated data from all participants collected during the MR measurements. In the bottom, the histogram shows the percentage of the total number of $\mathrm{G}+\mathrm{S}$ trials ( $n=2020$ ) sorted according to their measured SOA, showing how, as a result of the adaptive algorithm used, in the majority of trials the Gabors were flashed during the premotoric time window before saccadic onset. Only trials with SOAs between -100 and 0 were included in the analysis (gray bars and shaded area), whereas the rest (white bars) were discarded. The squares show performance in the orientation discrimination task. Percentage of trials in which subjects did not detect the presence of the Gabor stimuli is shown by the circles and indicates that, on $\sim 25 \%$ of the trials, the Gabors were not perceived, although they were presented on a stationary retina, a sort of saccadic blindness.

to $\sim 65 \%$, reaching chance levels $(50 \%)$ when presented intrasaccadically (Fig. 3). False alarms (defined by a button press on $\mathrm{S}$ trials) happened only rarely, in $<1 \%$ of the trials.

The introduction of a parametric modulator and secondorder polynomial expansion successfully explained additional variance in the BOLD signal extracted from the ROIs in all four subjects (subject BE, $t=2.77, p=0.002$; subject MK, $t=5.46$, $p<0.0001$; subject MT, $t=3.89, p<0.0001$; subject MU, $t=$ $4.74, p<0.0001)$. BOLD responses elicited by the condition $(\mathrm{G}+\mathrm{S})$ on trials with very long SOAs approximately correspond to the linear summation of the response amplitude elicited by the $\mathrm{G}$ and $\mathrm{S}$ conditions (supplemental Fig. 5, available at www. jneurosci.org as supplemental material) and systematically decreased as the onset of the Gabor stimuli approached the onset of the saccade (Fig. 4) (subject BE, $F=8.61$, df =3,4440, $p<0.001$; subject $\mathrm{MK}, F=21.14, \mathrm{df}=3,4440, p<0.0001$; subject $\mathrm{MT}, F=$ 22.62, df $=3,4440, p<0.0001$; subject $\mathrm{MU}, F=28.09$, $\mathrm{df}=$ $3,4440, p<0.0001)$. Comparison between Figures 3 and 4 shows how this decline in BOLD response parallels the decline in detection/discrimination performance observed in the behavioral task, starting up to $75 \mathrm{~ms}$ before the onset of any retinal motion.

\section{Discussion}

The behavioral data replicate a finding that is crucial for the interpretation of the imaging results: when presented immediately before the onset of the saccadic eye movement, Gabor stimuli are either not perceived at all or are perceived very poorly (Fig. 3). It is important to emphasize that this impairment occurs in the absence of any retinal motion, implying that the retinotopical correspondence is maintained for all analyzed trials.

Because of the slow temporal dynamics of the BOLD response, it is not possible to segregate the amount of signal change elicited by the Gabor stimuli from the signal produced by the gray background slipping along the retina immediately after the saccadic onset. To quantify the contribution from each of these two signal
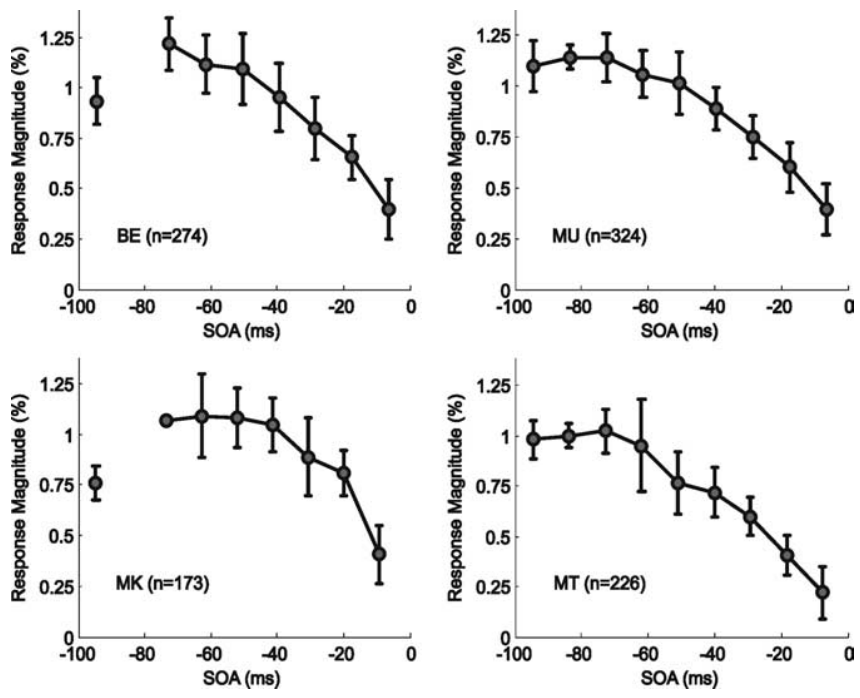

Figure 4. Average peak responses from $\mathrm{G}+\mathrm{S}$ trials as a function of $\mathrm{SOA}$. The amplitude of an otherwise constant hemodynamic response function was modulated by a second-order polynomial function to improve the fit to the data by accounting for the effect of SOA (see Materials and Methods, Data analysis). Each graph shows the average peak responses plotted against SOA across all 12 sessions for each of the four subjects. Error bars represent $\pm 1 S D$.

sources, conditions $S$ and $G$ were introduced in the experimental design as subcomponents of the $\mathrm{G}+\mathrm{S}$ condition ( $\mathrm{S}$ and $\mathrm{G}$ ) (supplemental Fig. 5, available at www.jneurosci.org as supplemental material), which can be considered as the sum of both. Worth noting is the fact that, despite the quantitatively smaller cortical responses measured in the $G$ trials, subjects are able to make accurate decisions on the orientation of four spatially distant Gabors, whereas larger responses triggered by saccadic motion on the S trials (supplemental Fig. 5, available at www.jneurosci.org as supplemental material) are perceptually disregarded. BOLD signals triggered by two stimuli occurring close in time have been shown to interact in a nonlinear manner (Huettel and McCarthy, 2000); nevertheless, no refractory effect of this kind could be found with interstimulus intervals shorter than $\sim 100 \mathrm{~ms}$, which is the latency of the evoked potential induced by the first of two pulsed stimuli (Ogawa et al., 2000). Thus, the decrease in BOLD signal observed in the encoding clusters when the Gabors were presented immediately before the onset of the saccadic eye movement are not likely to be related to some form of local nonlinear summation. Rather, the close temporal correspondence between behavioral and imaging data strongly suggests that the signal decline measured in the visual cortex is truly reflective of saccadic suppression. This decrease in the amplitude of the BOLD response could only have two origins. The first possibility is that feedback signals to V1 coming from higher visual or oculomotor cortical areas (e.g., signals from V4, V5, or the frontal eye fields) are centrally inhibited and that what we observe is actually a decrease in intracortical summation in V1 instead of an intrinsic decrease of V1 activity. These higher visual areas could interrupt feedback signals to V1 while oculomotor areas are engaged in the preparation of a saccade. Such an interruption of feedback processing could lead to a decline in conscious perception of the flashed stimuli, as evidenced by our psychophysical results (Fig. 3). An alternative explanation could be that saccadic suppression originates at a subcortical level, before retinal signals reach V1. Although both alternatives could account for our results, many recent physiological data converge around the latter.

Animal research has shown that the transmission of an evoked 
potential, induced by stimulation of the optic chiasm, through the LGN is reduced up to $60 \%$ during saccadic eye movements (Bartlett et al., 1976). In a similar experiment (Zhu and Lo, 1996), microelectrical stimulation of the superior colliculus consistently suppressed the evoked potential in the visual cortex by sending an inhibitory signal to the LGN. Reppas et al. (2002) measured the firing behavior of primate LGN neurons at time points before, during, and after horizontal saccades and observed a biphasic modulation of their firing rates, with a premotoric inhibition ( $\sim 50 \mathrm{~ms}$ before saccadic onset) followed by a strong enhancement after the saccadic offset. This effect was greater in neurons belonging to the magnocellular system.

Promising attempts to measure the overall modulation of saccade-related activity in human LGN and V1 have been made (Sylvester et al., 2005), reporting a reduction of the overall fMRI signal during saccades in both ROIs. These results, however, are difficult to interpret together with the physiological data, because BOLD signal changes triggered by the multiphasic responses observed by Reppas and colleagues in LGN will sum with unpredictable consequences within a blocked design. In a similar experiment from the same group (Sylvester and Rees, 2006), saccade-induced activation was found in LGN in the absence of visual input, whereas a suppression of activity was found to be dependent on the luminance level during visual stimulation in V1 for blocks of saccades compared with fixational periods. Because signal enhancement in the dark was also observed in V1 (although not significant), the authors suggest that this positive modulation (extraretinal in nature) could mask the suppressive effect of saccades on visually evoked responses to weak stimuli. In our study, the reduction in response magnitude never reaches the baseline level (as determined on the F trials). Although it is not possible to determine whether the suppression mechanism affects more strongly the G or the S signal component, based on the behavioral data, it seems reasonable to assume that at least a large part of this residual signal must be related to retinal motion induced by the saccade. Despite recent disagreement (Price et al., 2005), a possible explanation could be that the perceptual effect of saccadic image motion could be cancelled at a higher stage by populations of motion-sensitive neurons in medial temporal (MT/V5) (Thiele et al., 2002) and medial superior temporal cortex (Erikson and Their, 1991; Thiele et al., 2002) by selectively remaining silent or reversing its direction tuning during saccadic motion. Based on the above arguments, it can be concluded that saccadic suppression might actually be the result of at least two complementary processes: specific subcortical modulation and high-level motion canceling.

In summary, we combine retinotopic localization, high temporal resolution eye-tracking, event-related fMRI, and behavioral measurements to reveal the time course of saccadic suppression in human V1. Our data show how signals elicited by flashed stimuli are suppressed in primary visual cortex when presented immediately before a saccadic eye movement. Moreover, the present report demonstrates that, despite the sluggishness of the BOLD signal, this short-lived modulation, occurring within $100 \mathrm{~ms}$, can be resolved with fMRI. Our evidence indicates that an extraretinal saccadic suppression mechanism does exist in humans. Signals coming from the retina are suppressed every time a saccadic eye movement is performed, and this study demonstrates for the first time that cortical V1 responses to brief stimuli are greatly suppressed even when presented before the eyes begin to move.

\section{References}

Bartlett JR, Doty RW, Lee Sr BB, Sakakura H (1976) Influence of saccadic eye movements on geniculostriate excitability in normal monkeys. Exp Brain Res 25:487-509.

Brett M, Anton J, Valabregue R, Poline JB (2002) Region of interestanalysis using an SPM toolbox. NeuroImage 16:2.

Büchel C, Wise RJ, Mummery CJ, Poline JB, Friston KJ (1996) Nonlinear regression in parametric activation studies. NeuroImage 4:60-66.

Büchel C, Holmes AP, Rees G, Friston KJ (1998) Characterizing stimulusresponse functions using nonlinear regressors in parametric fMRI experiments. NeuroImage 8:140-148.

Burr DC, Morrone C (1994) Selective suppression of the magnocellular visual pathway during saccadic eye movements. Nature 371:511-513.

Castet E, Jeanjean S, Masson GS (2001) “Saccadic suppression": no need for an active extra-retinal mechanism. Trends Neurosci 24:316-318.

Diamond MR, Ross J, Morrone MC (2000) Extraretinal control of saccadic suppression. J Neurosci 20:3449-3455.

Engel SA, Glover GH, Wandell BA (1997) Retinotopic organization in human visual cortex and the spatial precision of functional MRI. Cereb Cortex 7:181-192.

Erikson RG, Their P (1991) A neural correlate of spatial stability during periods of self-induced visual motion. Exp Brain Res 86:608-616.

Friston KJ, Holmes AP, Poline JB, Grasby PJ, Williams SC, Frackowiak RS, Turner R (1995) Analysis of fMRI time-series revisited. NeuroImage 2:45-53.

Furmanski CS, Engel SA (2000) An oblique effect in human primary visual cortex. Nat Neurosci 3:535-536.

Garcia-Perez MA, Peli E (2001) Intrasaccadic perception. J Neurosci 21:7313-7322.

Huettel SA, McCarthy G (2000) Evidence for a refractory period in the hemodynamic response to visual stimuli as measured by MRI. NeuroImage 11:547-553.

Kleiser R, Seitz RJ, Krekelberg B (2004) Neural correlates of saccadic suppression in humans. Curr Biol 14:386-390.

Latour PL (1962) Visual threshold during eye movements. Vision Res 2:261-262.

Ogawa S, Lee TM, Kay A, Tank DW (1990) Brain magnetic resonance imaging with contrast dependent on blood oxygenation. Proc Natl Acad Sci USA 87:9868-9872.

Ogawa S, Lee TM, Stepnoski R, Chen W, Zhu XH, Ugurbil K (2000) An approach to probe some neural systems interaction by functional MRI at neural time scale down to milliseconds. Proc Natl Acad Sci USA 26:11026-11031.

Paus T, Marrett S, Worsley KJ, Evans AC (1995) Extraretinal modulation of cerebral blood flow in the human visual cortex: implications for saccadic suppression. J Neurophysiol 74:2179-2183.

Price NSC, Ibbotson MR, Ono S, Mustari MJ (2005) Reapid processing of retinal slip during saccades in macaque area MT. J Neurophysiol 94:235-246.

Reppas JB, Usrey WM, Reid RC (2002) Saccadic eye movements modulate visual responses in the lateral geniculate nucleus. Neuron 35:961-974.

Riggs LA, Volkmann FC, Moore RK, Ellicott AG (1982) Suppression of visual phosphenes during saccadic eye movements. Vision Res 22:423-428.

Ross J, Morrone MC, Goldberg ME, Burr DC (2001) Response: “saccadic suppression:” no need for an active extra-retinal mechanism. Trends Neurosci 24:113-121.

Sylvester R, Rees G (2006) Extraretinal saccadic signals in human LGN and early retinotopic cortex. NeuroImage 30:214-219.

Sylvester R, Haynes JD, Rees G (2005) Saccadic eye movements modulate visual responses in the lateral geniculate nucleus. Curr Biol 15:37-41.

Thiele A, Henning P, Kubischik M, Hoffmann KP (2002) Neural mechanisms of saccadic suppression. Science 295:2460-2462.

Thilo KV, Santoro L, Walsh V, Blakemore C (2004) The site of saccadic suppression. Nat Neurosci 7:13-14.

Volkmann FC, Schick AM, Riggs LA (1968) Time course of visual inhibition during voluntary saccades. J Opt Soc Am 58:562-569.

Wenzel R, Wobst P, Heekeren HH, Kwong KK, Brandt SA, Kohl M, Obrig H, Dirnagl U, Villringer A (2000) Saccadic suppression induces focal hypooxygenation in the occipital cortex. J Cereb Blood Flow Metab 20:1103-1110.

Zhu JJ, Lo FS (1996) Time course of inhibition induced by a putative saccadic suppression circuit in the dorsal lateral geniculate nucleus of the rabbit. Brain Res Bull 41:281-291.

Zuber BL, Stark L (1966) Saccadic suppression: elevation of visual threshold associated with saccadic eye movements. Exp Neurol 16:65-79. 